\title{
Variations in the Clinical Management of Multibacillary Leprosy Patients in Selected Hospitals in Metro Manila
}

\author{
Veincent Christian F. Pepito, ${ }^{1}$ Arianna Maever L. Amit, ${ }^{1}$ Rae Erica D. Samontina, ${ }^{1}$ \\ Sarah Jane A. Abdon, ${ }^{1}$ David Norman L. Fuentes ${ }^{1}$ and Ofelia P. Saniel ${ }^{2}$ \\ ${ }^{1}$ College of Public Health, University of the Philippines Manila \\ ${ }^{2}$ Department of Epidemiology and Biostatistics, College of Public Health, University of the Philippines Manila
}

\begin{abstract}
Introduction. This paper documents the variations in the diagnosis and management of multibacillary leprosy patients in three of the biggest case-holding hospitals in Metro Manila. Furthermore, we aimed to discuss the implications of these variations on the country's leprosy control and elimination program.
\end{abstract}

Methods. Focus group discussions (FGD) were conducted with 23 health professionals composed of doctors and nurses with at least a year of experience in managing leprosy patients. The topics included procedures on patient diagnosis and management such as treatment duration, patient follow-up and definitions of treatment completion and default. The FGD participants provided suggestions to improve treatment compliance of patients. Their responses were compared with World Health Organization (WHO) standards and/or the $2002 \mathrm{DOH}$ Manual of Operating Procedures (MOP) for leprosy. Transcripts of the recordings of the FGDs were prepared and thematic analysis was then performed.

Results. There were variations in the hospitals' procedures to diagnose leprosy, in treatment duration, and in patient follow-up. Definitions for treatment completion and default differed not just between hospitals but also with the WHO guidelines and the 2002 MOP. Hospitals extended treatment up to 24 or even 36 months, despite the 12 months stipulated in the MOP. Two hospitals required slit skin smear and skin biopsy in diagnosis, despite the MOP and WHO provisions that these were not mandatory. One hospital defined default as three consecutive months without treatment, which was different from the MOP and WHO standards and from the other hospitals.

Conclusion. Given the variations in patient management, we recommended that effectiveness of the standard treatment relative to other regimens being practiced by specialists be evaluated.

Key Words: Multibacillary Leprosy, Multiple Drug Therapy, Patient Management

\section{INTRODUCTION}

Parts of this research were disseminated via poster presentation in the 16th Department of Health - National Health Research Forum for Action at Sofitel Hotel, September 16, 2016, Pasay City, Philippines.

Corresponding Author: Veincent Christian F. Pepito College of Public Health

University of the Philippines Manila

625 Pedro Gil Street, Ermita Manila 1000 Philippines

Mobile Phone: +639065317664

Email:vcfpepito12345@gmail.com
Leprosy or Hansen's disease is a chronic infectious illness that affects the nerves and manifests as hypoaesthetic macules, papules, and lesions. The World Health Organization (WHO) classifies it into paucibacillary (PB), when there are five or less hypopigmented skin lesions, and multibacillary $(\mathrm{MB})$ when there are more than five of these skin lesions with invasion of the nasal mucosa. ${ }^{1}$ Patients with MB leprosy are treated with rifampicin, clofazimine, and dapsone for 12 months. Termed collectively as Multiple Drug Therapy (MDT), these medications are given monthly to patients in blister packs. ${ }^{1,2}$ 
In the Philippines, the prevalence of leprosy has dropped from 7.2 per 10,000 population in 1986 to 0.31 cases per 10,000 population by the end of 2010 and therefore has been eliminated as a public health problem. ${ }^{3,4}$ However, since pockets of cases still persist, eradication of the disease remains to be a challenge for the Department of Health (DOH). As of 2014, 90\% of the 2,771 reported leprosy patients in the country are MB. More than a fifth of these patients consult treatment facilities in Metro Manila. ${ }^{5}$

The Philippine National Leprosy Control Program developed a Manual of Procedures (MOP) adapted from WHO to standardize diagnosis and management of leprosy patients in the country. ${ }^{6}$ We hypothesized that there is non-compliance with the standard procedures among hospitals. Variations in patient management suggest the need to re-evaluate its effectiveness.

This paper aims to qualitatively document differences in diagnostic procedures, counselling, treatment initiation and monitoring of treatment compliance among three of the largest leprosy case-holding institutions in Metro Manila. In addition, this paper identifies notable variations in the definitions of treatment completion and default. Lastly, it offers some suggestions on how to improve leprosy control and treatment compliance, which will hopefully enhance government efforts towards the eradication of leprosy in the country.

\section{METHODS}

Health professionals with at least one year of experience in managing $\mathrm{MB}$ leprosy patients from selected hospitals in Metro Manila were invited to participate in focus group discussions (FGDs) conducted between February and June 2015. They were asked to share their usual procedures in managing leprosy patients. Specifically, they were asked regarding leprosy diagnosis, treatment duration, patient follow-up, and definitions of treatment completion and treatment default. Lastly, they were encouraged to provide suggestions to improve treatment compliance of leprosy patients. The topic guide used in the FGD is attached as a technical appendix in this paper.

The 'usual practices' of the health workers were compared with the 2002 National Leprosy Control Program (NLCP) $\mathrm{MOP}^{6}$ and/or Eighth Report of the WHO Expert Committee on Leprosy. ${ }^{7}$ At the time of the conduct of the study, these two documents contained the latest local and international standard guidelines for management of leprosy patients. The contents of the said documents, as well as the responses of medical personnel to the FGD questions, were organized according to themes and sub-themes. Thematic analysis was performed afterwards using QSR NVivo version 10 (QSR International, Melbourne, Australia).

Certificates of approval/clearance were obtained from the Ethics Review Committees of the participating hospitals and the institution prior to the conduct of the study.

\section{RESULTS}

Three nurses from Hospital A, five dermatology residents from Hospital $\mathrm{B}$, and 14 dermatology residents and one nursing assistant from Hospital C participated in three separate FGDs.

The participating hospitals are three of the largest leprosy case-holders in the entire National Capital Region. Hospital A, on average, treats 40 leprosy patients, Hospital B treats 64, while Hospital C treats around 100 patients each year. All three health institutions are government-owned but only Hospitals A and C are DOH-retained. ${ }^{a}$ Meanwhile, only Hospitals B and C are Philippine Dermatological Society (PDS) affiliated training hospitals for resident dermatologists.

The following paragraphs describe the usual procedures performed by health workers from the selected hospitals in managing leprosy patients. A summary of how the three hospitals manage their patients are shown in Table 1.

\section{Leprosy Diagnosis}

The 2002 NLCP MOP states that diagnosis of leprosy is made on the basis of clinical signs and symptoms, especially when there is history of contact with a positive case. The manual further emphasizes that rarely is there a need to do laboratory examination and other investigations to confirm a diagnosis. Slit-skin smear (SSS) examination is an optional diagnostic procedure that is performed when clinical diagnosis is doubtful. The main objective of the SSS is to ensure correct classification of a leprosy case as well as to confirm diagnosis. This is important so that the patient will be appropriately managed. ${ }^{6}$

Nurses from Hospital A suspect MB leprosy in a patient who has the characteristic lesions, infiltrations, deformities and nose collapse, among other features of the disease. When a suspected leprosy patient comes in for check-up, nurses ask the patient to put on a mask; this is to minimize the risk of transmitting the disease to other persons. Next, they assess the patient for numbness, lesions, and deformities. To confirm that the disease is indeed leprosy, they instruct the patient to undergo SSS examination and complete blood count (CBC) as part of the hospital's standard operating procedures (SOPs). Skin biopsy is rarely performed; however, it is only done when the initial SSS result is negative. SSS, CBC, skin biopsy and other laboratory tests relevant to the diagnosis and clinical management of leprosy are free of charge in Hospital A.

Resident doctors from Hospital B suspect MB leprosy in a patient who presents with hypoaesthetic plaques and papules. After noting patient history, they test for sensation through neurologic physical exams, such as monofilament test. When lesions are found to be hypoaesthetic, they perform SSS and skin biopsy as part of the protocol in Hospital B for

a $\mathrm{DOH}$ retained hospitals refer to all hospitals under the management and operation of DOH Center for Health Development. 
all suspected leprosy patients. As a last and crucial step, they do patient work-up to help decide if a patient should start treatment immediately.

Before 2013, only skin biopsy was part of Hospital B's SOPs in diagnosing leprosy patients. It was only later that SSS was included, after Hospital B doctors acknowledged its prognostic value. At present, specimen collection and processing are both done, without charge, in the hospital laboratory. However, reading and interpretation of test results are done outside the hospital for a fee. Doctors could waive this payment if the patient is indigent. Nevertheless, getting the smear read/interpreted outside the hospital may be inconvenient and costly for some patients so they opt not to have their SSS results read.

Dermatology residents from Hospital C suspect MB leprosy in a patient with erythematous patches and plaques. They consider the case as severe leprosy when a person presents with leonine facies and contracture of the extremities. Upon presentation, the doctors take the patient history. Afterwards, they perform physical and neurological examinations, SSS and skin biopsy. Results for the SSS are often available in the afternoon of the same day if the test was done in the morning; if not, the results are available the day after. Meanwhile it takes two weeks for skin biopsy results to be released.

CBC, SSS, skin biopsy, urinalysis, and liver and kidney function tests are performed for free in Hospital C. However, patients are required to pay for tests such as chest X-ray and glucose-6-phosphate dehydrogenase (G6PD) test.

\section{Treatment Initiation}

The 2002 MOP stipulates that treatment should start as soon as leprosy is confirmed in a patient. ${ }^{6}$

In Hospital A, nurses start treatment on the same day of diagnosis. However, some patients leave the hospital instead of waiting for their test results. This contributes to delay in treatment initiation.

Initiation of treatment in Hospital B is done on a caseto-case basis. Doctors start treatment right away in patients who manifest severe forms of leprosy such as madarosis or thinning of the eyebrows, and enlargement of ear lobules, even in the absence of confirmatory test results (i.e., SSS and skin biopsy). Unlike in Hospital A, Hospital B requires G6PD test to be done prior to starting the treatment. The doctors explained that G6PD assay is necessary to determine whether or not hemolytic anemia will occur when the patient takes in dapsone. They believe that having G6PD test will not cause a delay in initiation of treatment.

Similarly, doctors from Hospital C start treatment immediately only when the SSS result is positive. If it is found negative however, skin biopsy is done and a positive test result would indicate that treatment be started immediately. In these cases, treatment is said to start two weeks after the first consultation. G6PD test is a required procedure for leprosy patients in Hospital $\mathrm{C}$ as well.

\section{Counselling}

The WHO Expert Committee's report emphasizes the importance of counselling in addressing stigma of leprosy. ${ }^{7}$ The 2002 MOP, however, does not contain standards on the conduct of counselling for leprosy patients. Therefore, hospitals have different ways of counselling their patients as discussed in the succeeding paragraphs.

After diagnosis is confirmed, medical personnel of Hospital A ask patients regarding their knowledge of leprosy. Discussions about the disease, its manifestations, and its mode of transmission follow. In explaining the disease to the patient, health workers avoid using the term "ketong" the local name for leprosy - due to the stigma attached to it. Instead, they use the terms "Hansen's disease" or "leprosy". If relatives of patients are present, they are also invited to join the counselling session to encourage them to take part in the patient's treatment compliance and recovery. Two examples of how a nurse and a resident conduct counselling are provided below:

"I tell the patients that the disease is easy to cure. Just take the medications religiously. And after taking the medications for seven days, the bacteria are no longer as infective as before so as to spread the disease to those who live with you. But I also tell them the truth that before this check-up, everyone has already been exposed to you. There is a possibility that someone got infected, depending on his/her resistance. After that, I reiterate that after seven days, they are no longer infectious. This is important as their spouses are afraid of them."

- Nurse from Hospital A

"With regards to stigma, I personally avoid using the word 'ketong'. When I talk to the patient, I tell them you have Hansen's or leprosy'. If they hear the term 'ketong', their reaction is like, 'Ketong? Where did I get that?'. If you say it's Hansen's, it is like a kinder, gentler way of telling them what their illness is. And then if we explain that this is what is happening to you, this is your condition, they are more receptive of the diagnosis." - Dermatology resident from Hospital B

Meanwhile, resident doctors from Hospital C start counselling once SSS result is positive. In their sessions, they focus on the prognosis, as well as the consequences and implications of having the disease. In the hospital, counselling is said to be a long process that requires an entire day for new patients. However, for those who visit regularly, the process is relatively shorter. Counselling in this health institution usually begins with an explanation of the disease, its modes of transmission, and more importantly, its ability to infect other people in the household. Aside from introducing the patient to the disease, emphasis is placed on the necessity of treatment compliance and follow-up examinations once there are observable physiologic changes. 
"In counselling, we also stress the importance of compliance. They should also know the possible reactions to the drug because in leprosy, reactions are common because their immune system changes. (The message is that) even though they will have reactions, they should not stop taking the drug. Basically, we explain the treatment duration because it is for a year, for example, for multibacillary. So, we really explain that it is a long-term treatment, so you should followup with us. Because one of their problems is that when they experience contractures, they will no longer return for treatment. We always emphasize 'once you experience anything unusual, please come back and have yourself checked'"

\section{- Dermatology resident from Hospital C}

As part of counselling, flyers and pamphlets are given to leprosy patients in Hospitals A and B. In addition, the staff in the two hospitals supervise sessions provided by their respective Hansen's Clubs which allows: 1) counselling of several patients all at once; 2) supplementation of the usual doctor-patient counselling; and 3) interactions of patients with each other to share their experiences of living with the disease.

Hospital $\mathrm{C}$ has its own active leprosy support group that facilitates activities such as spiritual discussions, meetings and livelihood programs. However, its direct role in counselling was not explored during the FGDs.

\section{Patient Monitoring}

The 2002 NLCP MOP contains provisions for monitoring patients with special needs, as well as guidelines for monitoring clinical progress. For convenience and economy, patients who live far from the hospital are given more than a month's supply of medicines so that they need not come back often for their drugs: ${ }^{6}$

"... They should be given sufficient supply of the drugs to cover the period of absence. It is acceptable to give full course of treatment to these patients but they should be advised to report to the nearest rural health unit if they have complications."

- 2002 MOP p. 15

The MOP also stipulates that concerned health workers should note the following every time a patient returns for treatment: ${ }^{6}$

"Changes in the character of the lesion, pain in the eyes, changes in color of sclera and conjunctiva, new disabilities, or progression of previous disabilities, and nerve damage in the form of nerve pain, painless wounds or blisters, and difficulty in performing simple tasks like holding a pen, buttoning a shirt, or signs of any weakness or loss of prehensile skill."

- 2002 MOP p. 17
Nurses from Hospital A relate that MDT blister packs should be given monthly. A nurse recounted that she sends Short Messaging Service (SMS) messages to patients to remind them to come to the clinic to get their monthly MDT and for their regular checkup. Although this technique works to some extent, some patients do not give their true mobile numbers and therefore, cannot be contacted. Hospital A medical personnel also reported that some patients lie about their addresses so that they would not be located during contact tracing. The nurses attribute these infractions among the patients could be due to stigma attached to the disease.

"Like yesterday a neighbor of a patient approached me. The patient was already on treatment for eight months but eventually defaulted. Then the neighbor passed by the clinic. The neighbor asked if it was possible to ask for medications for the patient. I said, 'No, because the patient needs to be checked-up again'. The drug is for free, the patient just needs to come back."

- Nurse from Hospital A

There are cases in which the patient himself/herself requests for additional blister packs, presumably to minimize cost of transportation and long commute time. The nurses comply under reasonable circumstances (e.g. They gave 12 doses to an overseas contract worker assigned in Saudi Arabia); but they emphasize to patients that when lesions appear, they should come back to the hospital immediately to be checked by a health professional.

Doctors from Hospital B mentioned that it is ideal for patients to get the drugs monthly. However, they give leeway to patients who live far from the hospital or to those who live in areas where the health center does not have regular supplies of MDT blister packs. Nevertheless, they emphasized that patients who experience lepra reactions are asked to return every two weeks so that their motor-sensory functions can be monitored closely. Otherwise, they are expected to come only every 28 days. No standard protocols in the way patients are monitored and reminded for follow-up visits were mentioned during the FGD.

Resident doctors from Hospital C also allow some flexibility with patients who live far from the hospital and those who are indigent. However, only a maximum of three MDT blister packs, enough for three months, are provided. Exceptions to this policy, such as giving more than three blister packs to patients, are only done upon approval by the consultants.

Hospital $\mathrm{C}$ doctors check for lepra reactions, joint pains, and new lesions among patients who come for follow-up. When a patient is positive for lepra reactions, they ask him/ her to come back to the hospital every two weeks for close monitoring. Otherwise, they perform physical examination on the patient, especially the hands and feet, after which a nursing assistant gives him/her the MDT blister packs. They do not release a patient from treatment if he/she has had little 
change in his/her bacterial index from baseline after at least three months from completing treatment. The procedures for monitoring after this time period depends on the judgment of the consultant. Mechanisms on how patients are reminded for follow-up were also not mentioned during the FGDs.

\section{Treatment Completion}

The 2002 MOP defines treatment completion as having taken 12 MDT blister packs within 18 months for multibacillary cases. The manual further specifies that lesions may still appear even after treatment completion but these lesions will eventually resolve; thus, extension of treatment period is highly discouraged. ${ }^{6}$

Nurses from Hospital A classify their patients who have completed treatment into two: those who are 'released from treatment' and those who 'completed treatment'. According to them, a patient who is 'released from treatment' is a patient who has completed 12 MDT doses, is evaluated clinically, and is released from treatment by a doctor. On the contrary, a patient who 'completed treatment' is a patient who finished the prescribed number of MDT doses but did not return to the clinic for final evaluation.

There are patients in Hospital A and B whose treatment were extended up to 18 or even 24 months.

"For example, the patient has finished 12 blister packs and he/she underwent slit skin smear. On the basis of MI (morphological index) or BI (bacterial index), it was seen in the bacterial index that there is still 1\% alive (bacteria). (On this basis), the doctor will extend (the treatment) to 6 (more) months. Then after 6 months, if there are still viable bacteria detected, the bacterial index is still positive, (treatment will again be extended for another) 6 months, until 2 years. (After this, we are more) certain that the patient will be well by then." - Nurse from Hospital A

"We really do not follow the (WHO guidelines) very strictly. And as of this moment, I think the WHO already knows the stand of leprologists of the PDS (which is) once you are diagnosed with lepromatous leprosy, you should have 24 months of treatment, because if you complete 24 months of treatment, the risk of relapse is very low compared to those who only had 12 months of treatment. Once you give the reason or justify why you extended more than 12 months, it is alright with the WHO to release additional medications."

- Resident doctor from Hospital B

"The most common reasons why we extend treatment beyond 12 months are that patients did not have the desired amount of reduction in bacilli count at the end of 12 months, or that they still have clinicallyactive lesions, or they are experiencing reactions."

- Resident doctor from Hospital B
"Because there are also patients whom we treat as 'persisters.' The term 'persisters' means that even after two years of continuous MDT, these patients are still positive in slit skin or skin biopsy. From experience, I have a patient who was diagnosed in 2008 with Hansen's lepromatous. (From) 2008 to 2010, she had $M D T$ for two years. After two years of MDT, we repeated the tests to monitor her response to treatment; we performed slit skin and skin biopsy then we found out that there are still bacteria present. So, she continued treatment until 2012 when she was lost to follow up in 2012. Then she came back last year, around April, and then I repeated all the tests and still, bacteria were present."

$$
\text { - Resident doctor from Hospital B }
$$

Resident doctors from Hospital C reported that they make a distinction between 'released from treatment' and 'completed treatment', utilizing similar definitions with those provided by Hospital A nurses.

In Hospital A, nurses perform SSS at least twice: once at the start of treatment and a year later before the patient is released from treatment. SSS may be performed between these time periods as judged necessary by the doctor. Hospital B medical personnel perform SSS and skin biopsy to diagnose a patient. They then repeat every 12 months until the patient is released from treatment. Lastly, Hospital C residents perform SSS at the start of treatment and every six months thereafter until the patient is declared by the doctors to be done with the treatment. After 12 months, doctors check if the bacterial index is zero. If it is still positive but not exceeding 4+, treatment may be extended for up to six months. If it is at least 4+, treatment would be extended for another 12 months. They have recounted that some patients were treated for 36 months because SSS results contained viable bacteria even after 24 months of treatment.

\section{Treatment Default}

The 2002 MOP describes a defaulter as "a patient who has started treatment and who has not collected MDT drugs for six consecutive months" ${ }^{\prime 6}$ while current WHO definitions refer to a defaulter as "an individual who fails to complete treatment within the maximum allowed time-frame." Thus, an MB leprosy patient who misses six months of treatment is considered as a defaulter. ${ }^{7}$

Nurses from Hospital A said that they continue to use the definition of default as indicated in the 2002 NLCP MOP, despite getting a new report containing new definitions:

"We continue treating it as it is, but there's something in the manual right? In the manual, missing two months is already considered a default. Usually, it is two or three months. But the doctor said, just continue... (treating as if he/she did not default)."

- Nurse from Hospital A 
Furthermore, medical personnel from Hospital A confirmed that they currently have no definition of default for those who extend treatment.

Doctors from Hospital B adhere to the current WHO guidelines and emphasize that the six-month leeway may not be consecutive. They also apply these definitions to patients who have their treatment extended. This means that patients who are assigned to be treated for 18 months and 24 months have a maximum of 24 and 30 months, respectively, to complete the treatment.

Resident doctors from Hospital C said that they have more stringent criteria for default as suggested by their consultants. They said that if a patient does not come back for three consecutive months, the patient is labeled a defaulter. This definition also applies to patients whose treatment duration is extended.

\section{Recommendations by Health Workers}

This section presents recommendations suggested by participating health workers to improve treatment compliance of patients.

\section{Establishment of Health Centers and Making Treatment More Available}

Health care providers mentioned that their patients experienced shortages in medicines back in their places of residence, necessitating that they travel to Manila. The health professionals predict that this situation will adversely affect treatment compliance. To mitigate this, they suggested that more health centers with available MDTs be established so that patients will no longer have to travel to Metro Manila to get treated. Additionally, health workers advocated for the improvement of treatment availability (i.e. MDT and drugs for reactions), especially in hard-to-reach areas.

\section{Counselling and Support Groups}

Health workers recommended that the NLCP strengthen the implementation of its guidelines and focus training on counseling among frontline health workers. They emphasized that while trainings are usually about clinical diagnosis and management, training on counselling skills is equally important since this helps keep patients in treatment, assuage fears related to stigma, improve the patients' mental health status and improve their quality of life. ${ }^{7,8}$

Health workers also emphasized the importance of support groups and how incentives administered through these support groups could play a role in motivating patients to return for treatment.

\section{Active Case Finding and Health Education}

Health workers also recommended intensified case finding, especially in highly endemic areas.

Lastly, doctors emphasized the importance of health education for the reduction of stigma, which is a major hindrance to treatment compliance. They also suggested educating the patients, their families, and the community. Organizing events such as poster-making contests for grade school and high school students may help raise awareness.

\section{DISCUSSION}

This study has shown that there are major variations in the protocols for diagnosis, patient counselling, basis for initiating treatment, as well as patient monitoring. The hospitals included in the study used different definitions of 'treatment completion' and 'treatment default' and this could affect the way leprosy patients are managed.

Both the 2002 NLCP MOP and the latest report of the WHO Expert Committee on Leprosy do not require SSS and skin biopsy to be performed for diagnostic and monitoring purposes. ${ }^{6,7}$ However, the three hospitals require that these procedures be done. Health workers explained that SSS helps in determining prognosis and confirming diagnosis while skin biopsy is used in confirming leprosy when SSS result is negative. While the performance of more diagnostic tests increases certainty of diagnosis, this entails additional costs in terms of time, reagents and manpower. In addition to additional costs, the performance of skin biopsy might cause delays in the initiation of treatment and poorer compliance to doctor's orders, especially for Hospital B patients where skin biopsy is done outside the hospital.

Currently, there are no local guidelines for health workers in the counselling of leprosy patients. The suggestions put forth by some participants in the results section may be used in formulating these guidelines on counselling and in training of health workers in managing leprosy patients. Group counselling through leprosy support groups, can help minimize not only financial and temporal costs but also stigma as patients are given opportunities to interact with others. ${ }^{7,9,10}$

There are differences in how health workers monitor patient compliance to treatment. One nurse reminded patients through SMS messaging, only to be hindered by inaccurate contact details provided by the patients. As a result, patients failed to seek treatment, or discontinued treatment altogether. Several leprosy experts suggested that health workers should strive harder to increase the public's awareness and knowledge regarding the disease. ${ }^{7,911}$ Education of the family and the general public about leprosy will hopefully foster an atmosphere that encourages treatment compliance. While these efforts should continue, the importance of reminding patients to come back for treatment is paramount. Health workers dealing with leprosy patients can adopt good practices used in handling tuberculosis cases since both TB and leprosy require long periods of treatment. Regularly reminding patients to come for treatment appeared to have worked well in TB control. ${ }^{12}$

Resident physicians discussed the need for extending treatment on certain occasions. They extend it to a maximum of 36 months, far from the 12 months duration suggested by the WHO. Some medical personnel say that 24 months 
of treatment is effective for patients with an initial bacterial index of $4+$ or higher. This is recommended by the PDS and is supported by the Guidelines for the Control of Leprosy in the Northern Territory of Australia. ${ }^{13}$ These state that extending treatment to 24 months in patients with an initial bacterial index of at least $4+$ or higher minimizes relapse. ${ }^{13-15} \mathrm{~A}$ doctor from one of the hospitals also elaborated on the presence of 'persisters' as a reason for extending treatment. 'Persisters' have been defined as "permanently or partially dormant organisms that have the capacity to survive in the host despite adequate chemotherapy." ${ }^{16}$ Its presence is thought to be a risk factor for relapse once activated, and a few of these 'persisters' have been documented to be resistant to dapsone and rifampicin. ${ }^{16,17}$

These findings have wide-ranging implications in terms of cost and duration of treatment. Extending treatment might decrease the risk of relapse, but this will entail more costs. Furthermore, while there are studies documenting relapses after MDT, ${ }^{15-18}$ there is no evidence in literature to support the belief that the 24-month duration is indeed cost-effective in preventing relapse among leprosy patients. As such, further studies are needed to establish clear-cut policies with regard to treatment duration. Some issues, such as the emergence of 'persisters' and drug-resistant leprosy, should be addressed alongside those concerning treatment duration. Policies that will benefit patients the most must be crafted to prevent the further spread of leprosy.

There were issues with the hospitals' definitions of default. This means that defaulter statistics from each hospital may not be comparable. Thus, definitions of default, as well as for those patients who were placed on extended treatment, should be standardized as they may represent substantial number of patients in treatment.

Among the various practices mentioned, none is considered inferior or superior to another. As such, the results of this study should not be utilized to incriminate hospitals for not observing the treatment guidelines. Rather, these differences in practices should be assessed through further research to determine which practice or combinations of practices, given constraints in time, manpower and resources, will contribute most in efforts towards elimination of the disease.

\section{CONCLUSIONS}

There are variations in the clinical management of leprosy patients between the three hospitals, and from Philippine NLCP MOP, and the WHO standards. These variations may necessitate their evaluation relative to the standard treatment to determine the most effective way to manage leprosy patients.

\section{RECOMMENDATIONS}

The following recommendations are made by the researchers to address the issues highlighted in the study.

\section{Standardize definitions of treatment completion and default}

The definitions of treatment completion and default should be standardized and used consistently in all treatment centers to allow accurate computation of treatment completion and defaulter rates as well as allow comparability of information. Standardizing definition of terms and patient management will allow more valid statistics to monitor progress towards disease elimination.

\section{More in-depth training of health professionals which includes psychological evaluation of patients and counselling}

Often an underrated aspect of case management of leprosy, counselling was deemed by the healthcare providers as a vital aspect in patient treatment compliance. In addition to establishing guidelines for counselling of leprosy patients, healthcare providers should also be trained in psychological evaluation of patients and counselling. This will help in assuaging patient fears related to stigma, and hopefully, in improving treatment compliance.

\section{Further Research}

This study highlights the need to further evaluate the effectiveness of current practices of healthcare providers relative to the current standards of leprosy patient management.

\section{Technical Appendix - FGD Questionnaire}

Good Morning/Afternoon/Evening Sir/Ma'am! Today we will talk about the reasons for treatment compliance and default of leprosy patients from your experience as a leprosy worker and about the MDT therapy. Please do answer the questions honestly and with the best of your abilities. We cannot give you full assurance that the information that you gave will be kept confidentiality since you are joined by others in this FGD. Can we begin the FGD now?

1. Can you describe what you usually do when a suspected leprosy patient comes in for consultation?

2. Can you please describe to us how a person with leprosy looks like?

3. What clinical or lab tests do you usually do in the hospital to diagnose a suspected patient?

3.1 Were there instances when the clinical or lab tests could not be done on the patient? Why does this happen?

4. What do you usually do to confirm the initial diagnosis? 4.1 Ask for reasons why no confirmation is done if this is the case. What do you usually recommend your patients do if diagnosis cannot be confirmed?

5. What do you tell the patient after he/she is diagnosed?

5.1 Probe to determine if counseling is done.

5.2 Find out how counseling can affect treatment compliance. 
6. When do you usually start MDT?

6.1 If patients are not placed on MDT immediately, ask for the reasons.

6.2 If patient is referred to another facility, ask why this is the case.

7. For time that you have worked with leprosy patients, have you encountered patients who did not finish the treatment prescribed (default cases)?

7.1 If yes, why do you think they defaulted?

8. For patients who fully complied with their treatment regimen, what do you think are their reasons for doing so?

9. How do you do follow-up your patients?

10. In what way will disability and other complications influence treatment compliance? In what way can stigma due to the disease affect treatment compliance?

11. What can you suggest to improve treatment compliance to the MDT?

Thank you for your time.

\section{Acknowledgments}

The authors express their heartfelt gratitude to the University of the Philippines Manila - National Institutes of Health for funding the study through the University of the Philippines Manila Student Researcher Grant. The authors would also like to thank the health professionals who participated in this study for their insights regarding leprosy control in the country.

\section{Statement of Authorship}

All authors approved the final version submitted.

\section{Author Disclosure}

All authors declared no conflict of interest.

\section{Funding Source}

The project was partially funded by the University of the Philippines Manila Student Researcher Grant 2014 (Project Code: 2014-046)

\section{REFERENCES}

1. World Health Organization. Epidemiological Review of Leprosy in the Western Pacific Region 2008-2010: Sustaining Leprosy Services and Further Reducing the Leprosy Burden. Manila: World Health Organization Western Pacific Regional Office; 2011.

2. World Health Organization. Enhancing Global Strategy for Further Reducing the Disease Burden Due to Leprosy. New Delhi: World Health Organization South East Asia Regional Office; 2009.

3. Gajete F, Costo E, Merla M, Enriquez R. Leprosy Control and the Burden of Leprosy in the Philippines: 2006-2010. Manila: Department of Health (Philippines), National Leprosy Control Program.

4. Handog E, Gabriel M, Co C. Leprosy in the Philippines: a review. Int J Dermatol. 2011;50(5):573-81. doi: 10.1111/j.13654632.2011.05044.x.

5. Villalon E, National Leprosy 2013 Annual Statistical Report. Manila: Department of Health (Philippines), National Leprosy Control Program; 2014.

6. Department of Health (Philippines). National Leprosy Control Program 2002 Manual of Operations. Department of Health (Philippines), National Leprosy Control Program; 2002.

7. Al-Qubati Y, de Oliveria M, de Palma Caldas M, Fine P, Gupte M, Kawuma H , et al. WHO Expert Committee on Leprosy: Eighth Report. ISSN: 0512-3054. Geneva: World Health Organization; 2012.

8. Tsutsumi A, Izutsu T, Islam A, Maksuda A, Kato H, Wakai S. The quality of life, mental health, and perceived stigma of leprosy patients in Bangladesh. Soc Sci Med. 2007;64(12):2443-53

9. Rafferty J. Curing the stigma of leprosy. Lepr Rev 2005; 76:119-126

10. Floyd-Richard M, Gurung S. Stigma reduction through group counselling of persons affected by leprosy - a pilot study. Lepr Rev. 2000;71(4):499-504.

11. van den Broek J, O'Donoghue J, Ishengoma A, Masao H, Mbega M. Evaluation of a sustained 7-year health education campaign on leprosy in Rufiji District, Tanzania. Lepr Rev. 1998;69(1):57-74.

12. Liu Q, Abba K, Alejandria M, Sinclair D, Balanag M, Lansang M Reminder systems to improve patient adherence to tuberculosis clinic appointments for diagnosis and treatment. Cochrane Database Syst Rev. 2014;(11):CD006594. doi: 10.1002/14651858.CD006594.pub3.

13. Coates E, Judd J, Gisjbers K, Scott L, Krause V. Guidelines for the Control of Leprosy in the Northern Territory 2010. Casuarina: Department of Health and Families, Center for Disease Control.

14. Malathi M, Thappa D. Fixed-duration therapy in leprosy: limitations and opportunities. Indian J Dermatol. 2013; 58(2):93-100.

15. Balagon M, Cellona R, dela Cruz E, et al. Long-term relapse risk of multibacillary leprosy after completion of 2 years of Multiple Drug Therapy (WHO-MDT) in Cebu, Philippines. Am J Trop Med Hyg. 2009;81(5):895-9. doi: 10.4269/ajtmh.2009.09-0189.

16. Kaimal S, Thappa D. Relapse in Leprosy Indian J Dermatol Venereol Leprol. 2009;75(2):126-35

17. Poojabylaiah M, Marne R, Varikkodan R, Dandakeri S, Bala N, Martis J. Relapses in multibacillary leprosy patients after multidrug therapy. Lepr Rev. 2008; 79(3):320-24 .

18. Desikan K, Sundaresh P, Tulasidas I, Rao PV. An 8-12 year follow-up of highly bacillated Indian leprosy patients treated with WHO multidrug therapy. Lepr Rev. 2008;79(3):303-10. 


\section{APPENDIX}

Table 1. Summary of practices of the various hospitals in terms of diagnosis, treatment initiation, counselling, patient monitoring, treatment completion, and default as compared to the 2002 DOH Leprosy MOP and/or the WHO Expert Committee Report.

\begin{tabular}{lll} 
& 2002 DOH Leprosy MOP ${ }^{6}$ or the & \multicolumn{1}{c}{ Hospital A } \\
Diagnosis & MOP: Diagnosis of leprosy & Assessment of signs and \\
& is based on clinical signs and & symptoms. \\
symptoms; especially when & SSS and complete blood count \\
there is history of contact with & (CBC) is required. \\
positive case. & Skin biopsy is only performed if \\
Slit skin smear (SSS) is only & SSS is negative. \\
performed when diagnosis & Laboratory tests relevant to \\
is doubtful. & diagnosis of leprosy is free.
\end{tabular}

\section{Hospital B}

Assessment of signs and symptoms; including neurologic physical exams. Specimen collection for SSS and skin biopsy is required and is performed for free at the hospital. Slit skin smear readings are done outside the hospital and may not be free.

\begin{tabular}{lll}
\hline $\begin{array}{l}\text { Treatment } \\
\text { Initiation }\end{array}$ & $\begin{array}{l}\text { MOP: Treatment should start as } \\
\text { soon as leprosy is diagnosed in } \\
\text { a patient. }\end{array}$ & $\begin{array}{l}\text { Treatment started on the same } \\
\text { day of diagnosis. }\end{array}$
\end{tabular}

Counselling MOP: Not discussed.

WHO Expert Committee Report: Counselling is important in addressing stigma of leprosy, and should be started at the time of diagnosis.

Initiation of treatment is

done on a case-to-case basis. G6PD test is required before treatment is started. Counselling on what the disease is about, its manifestations, and its mode of transmission. Emphasis is given on avoiding stigmatizing terms in disclosing the diagnosis

\section{Counselling on what the disease is about, its manifestations, and its mode of transmission. Emphasis is given on the benefits of treatment. Relatives are included in counselling if they are present. Flyers and pamphlets are given. Group counselling through Hansen's clubs.} to the patient. Flyers and pamphlets are given. Group counselling through Hansen's clubs.

\begin{tabular}{lll}
\hline Patient & MOP: Patients may be given extra & Use of Short Messaging Service \\
Monitoring & $\begin{array}{l}\text { medicines so that they need to } \\
\text { come back often if they live far } \\
\text { from the hospital. }\end{array}$ & $\begin{array}{l}\text { (SMS) to remind patients to get } \\
\text { their monthly treatment and } \\
\text { check-up. }\end{array}$
\end{tabular}
Health workers should observe changes to the character of the lesions, and development of new signs or symptoms.

Treatment MOP: Treatment completion is Completion defined as "having taken 12 MDT blister packs within 18 months".

\begin{tabular}{ll}
\hline Treatment & MOP: Having started treatment \\
Default & but has not collected MDT drugs \\
& for six consecutive months"
\end{tabular}

WHO Expert Committee Report: An individual who fails to complete treatment within the maximum allowed time frame.

\section{Giving more than a month's} treatment is allowed under certain circumstances.

\section{Patients who have completed}

treatment is classified into two:

"released from treatment" and

"completed treatment".

Treatment may be extended up to 18 or even 24 months depending on the bacterial or morphological index.

SSS is performed at least twice: at the start of treatment and before the patient is released from treatment. It may also be performed any time at the discretion of the doctor.

\section{Mentioned a new report where} two consecutive months of not getting treatment is already considered default.

There is no definition of default for those who extended treatment.
Giving more than a month's treatment is allowed under certain circumstances. Patients who experience lepra reactions are asked to return every two weeks.

\section{Treatment may be}

extended up to 18 or even

24 months depending on the bacterial or morphological index. SSS and skin biopsy is performed at diagnosis and is repeated every 12 months until the patient is released from treatment.
Hospital C

Assessment of signs and symptoms; including neurologic examinations.

SSS and skin biopsy are required tests; CBC, SSS, skin biopsy, urinalysis, and liver and kidney function tests are free, but chest $X$-ray and glucose-6phosphate dehydrogenase test (G6PD) are not. Treatment is only initiated if SSS or skin biopsy is positive. G6PD test is also required.

Counselling on what the disease is about, its manifestations, mode of transmission.

Emphasis is given on prognosis, consequences of having the disease, and the importance of treatment compliance and follow-up examinations. Has active leprosy support groups. Maximum of three blister packs are given to a patient. Lepra reactions, joint pains and new lesions are observed every check-up. Patients who experience lepra reactions are asked to return every two weeks.

Patients who have completed treatment is classified into two: "released from treatment" and "completed treatment". Treatment may be extended up to 36 months because SSS results contained viable bacteria even after 24 months of treatment. SSS is done at the start of treatment and every six months thereafter until completion of treatment.

A patient is considered defaulted if they do not come back for three consecutive months.
Six months' leeway may not be consecutive for a patient to be considered defaulted. This definition is also applied to those who have extended treatment. 